\title{
Propriedades de ordenamento do modelo votante em redes de escala livre
}

\author{
(Ordering properties of the voter model on scale-free networks)
}

\author{
Reginaldo A. Zara e Elizabete Koehler \\ Universidade Estadual do Oeste do Paraná, Centro de Ciências Exatas e Tecnológicas, \\ Cascavel, Paraná, Brasil.
}

Recebido em 21/11/03; Aceito em 12/3/04

\begin{abstract}
O modelo votante de 2 estados pode ser utilizado na introdução dos princípios básicos das simulações de Monte Carlo de sistemas físicos. Neste trabalho realizamos simulações de Monte Carlo do modelo votante de 2 estados definido sobre redes de escala livre. Verificamos que o sistema exibe um regime transiente cuja duração depende do tamanho da rede sugerindo que, assintoticamente, o sistema não converge para um estado absorvente. Por outro lado, em redes de tamanhos finitos, o sistema permanece em um estado ativo durante um tempo $\tau$ após o qual converge para um estado totalmente ordenado, sendo que este tempo médio de permanência em um estado ativo aumenta com o tamanho da rede. Nossos resultados estão de acordo com as simulações do modelo votante em redes Small World apresentadas recentemente por Vespignani et al.
\end{abstract}

Palavras-chave: sistemas de spins, redes complexas.

The 2-state voter model may be used to introduce the Monte Carlo simulations of physical systems. In this work, we performed Monte Carlo simulations of the 2-state voter model on scale-free networks. We found that in finite size networks, the system converges to an ordered state. Nevertheless, in the thermodynamic limit the systems do not exhibit completely ordered states, being settled in a transient state with coexisting opinions. Our results agree with the simulations of the voter model on Small World Networks presented by Vespignani et al.

Keywords: spins systems, complex networks.

\section{Introdução}

A simulação de Monte Carlo é uma técnica amplamente utilizada na investigação de fenômenos que envolvem comportamento coletivo de partículas [1]. Um exemplo comum é o estudo da dinâmica do ordenamento de sistemas magnéticos em alta temperatura subitamente resfriados à temperatura zero [2,3]. Atualmente, as idéias utilizadas no estudo de sistemas magnéticos têm sido adaptadas à investigação de fenômenos sociais como o estabelecimento de consenso em torno de uma opinião [4,5], formação de estruturas hierárquicas em sociedades [4,5], distribuição de votos em eleições [6,7], etc. Diversos modelos para dinâmica de opiniões têm sido propostos e sistematicamente investigados através da aplicação de diferentes métodos [7-10]. As simulações computacionais têm sido utilizadas para investigar o comportamento de sistemas que, partindo de uma distribuição aleatória de opiniões, evolui segundo um modelo dinâmico, atingindo estados finais de consenso ou de diversidade de opiniões.

Possivelmente o modelo mais simples para descrever a troca de opiniões em uma população seja o modelo votante de 2 estados [11,12]. Este modelo é análogo a um ferromagneto em temperatura zero. Assim, partindo de uma configuração inicial desordenada, espera-se que o sistema evolua para uma configuração final onde todos os spins apontam na mesma direção. Usualmente o modelo votante é definido em uma rede regular de dimensão d. Neste caso,

Enviar correspondência para Reginaldo A. Zara. E-mail: razara@ unioeste.br assume-se que cada sítio da rede é caracterizado por uma variável de spin $(s=+1$ ou $s=-1)$ representando duas opiniões diferentes. Partindo de uma configuração totalmente desordenada, onde metade da população tem opinião semelhante (e contrária a da outra metade), simula-se a situação em que, depois da quebra de simetria, a configuração de spins deverá evoluir para uma única opinião (correspondente à fase ferromagnética). No modelo votante de 2 estados o sistema evolui através de uma regra dinâmica simples: cada sítio da rede é consultado e assume o estado de um de seus vizinhos, escolhido aleatoriamente. Obviamente, pode-se questionar o fato de que um indivíduo aceita (sem nenhuma exigência) a opinião de seu vizinho, porém, ressaltamos que este é o modelo mais simples possível, e que pode ser enriquecido adicionando-se vínculos e restrições, como a probabilidade de que a opinião do vizinho não seja aceita, a consulta a todos os vizinhos, a utilização de uma regra de maioria, etc. Em redes regulares, as propriedades do modelo votante são bastante estudadas $[11,12]$. Especificamente, em redes com dimensão $d=1 \mathrm{e}$ $d=2$, o modelo votante de confrontação de pares evolui convergindo para uma configuração na qual todos os sítios assumem a mesma variável de estado. Como o sistema não pode escapar desta configuração, temos um estado absorvente $[11,12]$.

Recentemente, Vespignani et al. [13,14] investigaram o modelo votante definido em uma rede Small World $[15,16]$. Seus resultados indicam que as conexões de longo alcance afetam o processo de ordenamento de maneira não trivial, inibindo o ordenamento e sugerem que, mesmo no 
limite termodinâmico, o sistema não converge para uma configuração absorvente. Por outro lado, quando a rede possui um número finito de sítios, observa-se que o ordenamento total do sistema ocorre em tempos mais curtos do que aqueles observados para redes regulares de mesmo tamanho.

Neste trabalho simulamos o modelo votante definido sobre as redes de escala livre resultantes do modelo de crescimento de Barabási-Albert [17]. As redes construídas segundo o algortimo de Barabási-Albert resultam de um modelo de crescimento preferencial no qual cada novo sítio incorporado à rede conecta-se preferencialmente aos sítios que possuem maior grau de conectividade. Nossos resultados revelam que as interações de longo alcance presentes nas redes de escala livre inibem o ordenamento completo do sistema e tornam os efeitos de tamanho da rede muito mais pronunciados do que aqueles observados por Vespignani et al. nas redes Small World.

\section{Simulação e resultados}

O modelo votante de 2 estados definido em redes regulares têm sido bastante estudado, tanto analiticamente quanto através de simulações computacionais. Desta forma, muitas de suas propriedades são bem conhecidas $[11,12]$. Neste trabalho investigamos, através de simulações de Monte Carlo, o modelo votante definido sobre redes não regulares, em especial, nas redes de escala livre.

Recentemente, Vespignani et al. [13] simularam o modelo votante em redes Small World, comparando seus resultados com o comportamento do modelo definido em uma rede unidimensional com interações entre primeiros e segundos vizinhos. Para a construção da rede Small World, Vespignani et al. utilizaram uma rede unidimensional, com condições periódicas de contorno e considerando conexões entre primeiros e segundos vizinhos. O estabelecimento de conexões de longo alcance segue o algoritmo padrão de Watts-Strogatz: a ligação que une o sítio $i$ ao sítio $j$ é consultada e transferida, com uma probabilidade $p$, para algum sítio $l \neq j$ escolhido aleatoriamente. Variando o parâmetro $p$ no intervalo $[0,1]$, pode-se interpolar entre redes unidimensionais $(p=0)$ e grafos aleatórios. Embora este procedimento introduza conexões de longo alcance, o número total de ligações na rede permanece constante. Neste caso específico, a exemplo da rede unidimensional com ligações entre primeiros e segundos vizinhos, os sítios da rede Small World possuem um número médio de ligações $<k>=4$.

Para simular o modelo votante em uma rede de escala livre, construímos uma rede de sítios interagentes utilizando o modelo de Barabási-Albert [17]. O algoritmo de crescimento de uma rede de escala livre de Barabási-Albert utiliza como ingredientes principais o crescimento da rede e vinculação preferencial de sítio e pode ser descrito da seguinte forma. Começamos com um número inicial de sítios fixo e aleatoriamente conectados entre si. Em cada instante de tempo um novo sítio é adicionado à rede, vinculando-se a $m$ diferentes sítios já presentes na rede com uma probabilidade $p$ não uniforme. A probabilidade de que o novo sítio vincule-se a um sítio $i$ já presente na rede é dada por

$$
p\left(k_{i}\right)=\frac{k_{i}}{\sum_{j} k_{j}}
$$

onde $k_{i}$ é o número de conexões que o sítio $i$ possui, enquanto a soma é efetuada sobre todos os sítios da rede. Esta equação reflete a hipótese de que os sítios mais conectados possuem maior probabilidade de vinculação. O crescimento ocorre até que a rede atinja uma quantidade total $N$ de sítios. Independentemente do valor de $m$, as distribuições de conectividades decaem segundo leis de potências $\left(P(k) \sim k^{-\gamma}\right)$, traço característico das redes de escala livre. Para uma rede de escala livre formada por $N$ sítios, o número médio de conexões de cada sítio é $<k>=2 m$ [17].

Após o processo de preparação das redes iniciamos a simulação de Monte Carlo para o modelo votante. Atribuímos a cada sítio da rede uma variável de estado $s=+1$ ou $s=-1$ com igual probabilidade, ou seja, partimos de uma configuração inicial totalmente desordenada. Em seguida aplicamos as regras de atualização: Cada sítio $i$ da rede é consultado e seu estado é confrontado com o estado um de seus vizinhos $j$ escolhido aleatoriamente. Se $s_{i} \neq s_{j}$ o sítio $i$ adota o estado do sítio $j$, ou seja, fazemos $s_{i}=s_{j}$. Após todos os sítios da rede terem sido consultados temos um passo de Monte Carlo. As regras de atualização são aplicadas durante t passos de Monte Carlo.

Em nossas simulações avaliamos duas quantidades: a fração de ligações ativas $N_{A}$ (definida como a razão entre o número de conexões entre sítios com diferentes estados e o total de conexões da rede) e o tempo durante o qual existem ligações ativas na rede, o qual denominamos tempo de sobrevivência $(\tau)$. A fração de ligações ativas está relacionada ao tamanho médio dos domínios formados no sistema. Em geral podemos relacionar o tamanho médio dos domínios com a quantidade $1 / N_{A}$. Assim, quando a quantidade $N_{A} \rightarrow 0$ o sistema converge para um estado absorvente. $\mathrm{O}$ tempo de sobrevivência nos fornece o número médio de passos de Monte Carlo necessário para que o sistema atinja o estado absorvente.

Na rede regular unidimensional a fração de ligações ativas decai com o tempo segundo uma lei de potências $\left(N_{A} \sim t^{-1 / 2}\right)$, ou seja, temos um processo de relaxação para um estado absorvente. Quando interações de longo alcance são introduzidas na rede, o sistema muda de comportamento. Segundo Vespignani [13], a análise da fração de ligações ativas revela que, no limite termodinâmico, a rede Small World permanece em um estado de totalmente desordenado, resultando em um conjunto de domínios de tamanho aproximadamente constante. Em sistemas de tamanho finito, após um regime de coexistência de estados o sistema converge exponencialmente para uma configuração absorvente. Observa-se que este ordenamento total do sistema ocorre em tempos mais curtos do que aqueles observados para redes regulares de mesmo tamanho. Além disso, o tempo necessário para o ordenamento aumenta com o número de sítios da rede segundo uma lei de potências. 
Para comparação simulamos o modelo votante definido sobre de redes de escala livre com $m=2$, o que leva a redes de conectividade média $\langle k\rangle=4$. Na Fig. 1 temos o gráfico da fração de ligações ativas como função do tempo para as redes Small World e de escala livre compostas pelo mesmo número de sítios (e mesma conectividade média). Em ambos os tipos de rede o sistema evolui exibindo um regime transiente cuja duração aumenta com o número de sítios da rede. Durante sua permanência no regime transiente, domínios de diferentes estados coexistem. Porém, nas redes de escala livre o tamanho médio destes domínios $\left(1 /\left(N_{A}\right)\right)$ é menor do que aquele observado para as redes Small World. Atribuímos a coexistência de domínios de diferentes estados às propriedades de conectividade da rede, uma vez que, embora a conectividade média das redes sejam equivalentes suas distribuiçõos de conectividades são bastante diferentes. A distribuição de conectividades da rede Small World é do tipo exponencial e, com isso, a maioria dos sítios possuem conectividade em torno do valor médio $<k=4>$, ou seja, a presença de sítios com elevado grau de conectividade é rara. Para as redes de escala livre as distribuições de conectividades seguem leis de potência. Isto implica na abundância de sítios de baixo grau de conectividade e uma minoria estatisticamente significativa de sítios de elevado grau de conectividade. Estes sítios, que interagem com muitos outros sítios através de conexões de longo alcance, funcionam como obstáculos para a difusão livre das fronteiras dos domínios, dificultando a fusão entre os domínios e consequentemente o ordenamento do sistema. Como podemos observar na Fig. 1, após o regime transiente, os sistemas convergem rapidamente para um estado absorvente, sendo que o tempo de permanência no estado transiente, é menor para as redes de escala livre. No inset da Fig. 1 vemos que o processo de evolução para o estado absorvente (totalmente ordenado) é nitidamente exponencial. Na Fig. 2 a temos fração de ligações ativas como função do tempo em redes de escala livre de diferentes tamanhos e $m=2$. Como pode ser observado, durante a evolução temporal, sistemas de tamanho finito permane-

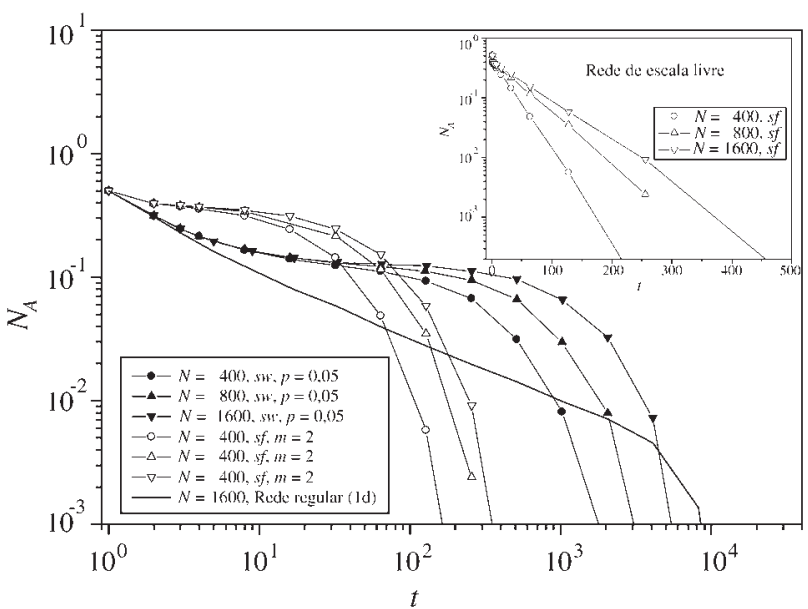

Figura 1 - Fração de ligações ativas como função do tempo para redes Small World $(s w)$ e de escala livre $(s f)$ formadas pela mesma quantidade de sítios. A quantidade $\left(1 /\left(N_{A}\right)\right)$ é proporcional ao tamanho médio dos domínios. Inset: $\mathrm{O}$ grafico de $N_{A}$ como função do tempo para redes de escala livre em escala mono-log revela que o processo de evolução para o estado ordenado é exponencial.

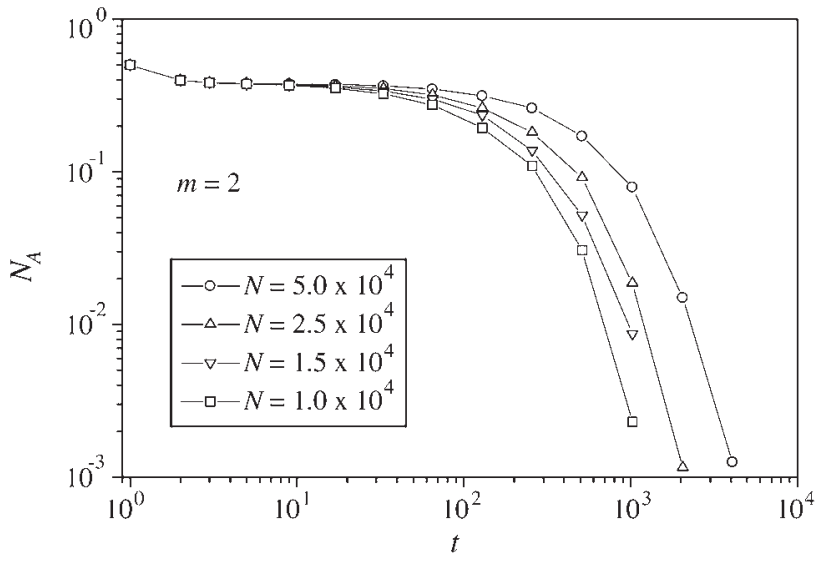

Figura 2 - Fração de ligações ativas com função do tempo para redes de escala livre $(m=2)$ e diferentes número de sítios.

cem em um regime transiente com atividade constante durante um certo intervalo de tempo, quando então, devido grandes flutuações espontâneas, convergem exponencialmente para uma configuração estável (estado absorvente). Podemos perceber também que o tempo de permanência em um estado ativo, denominado tempo de sobrevivência $(\tau)$, cresce com número de sítios da rede, sugerindo que nas redes de escala livre formada por um número muito grande de sítios, o sistema permanece em um estado completamente desordenado.

Na Fig. 3, mostramos o comportamento da fração de ligações ativas como função do tempo para $N=25000$ e diferentes valores de $m$. Podemos observar que, independentemente da conectividade, o sistema exibe o mesmo tipo de comportamento, ou seja, permanece em um estado transiente desordenado durante um certo intervalo de tempo, convergindo posteriormente para um estado absorvente. Esta figura também sugere que o tempo de sobrevivência cresce com a conectividade da rede.

No tratamento das redes de escala livre, a extrapolação para o limite $N \rightarrow \infty$ deve ser tratada com cuidado. Para o modelo de Barabási-Albert, a distribuição de conectividades segue uma lei de potências $P(k) \sim k^{-3}$ e, com isso, se o número de sítios for infinito, a conectividade média $(<k>)$ diverge. Assim, de acordo com a Fig. 3, que o tempo

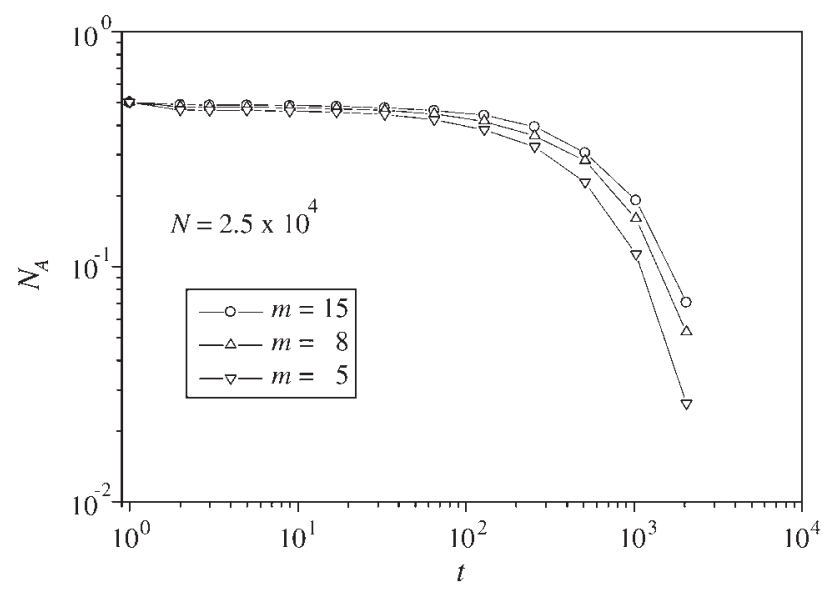

Figura 3 - Fração de ligações ativas com função do tempo para redes de escala livre $N=25000$ e diferentes valores de conectividade. 


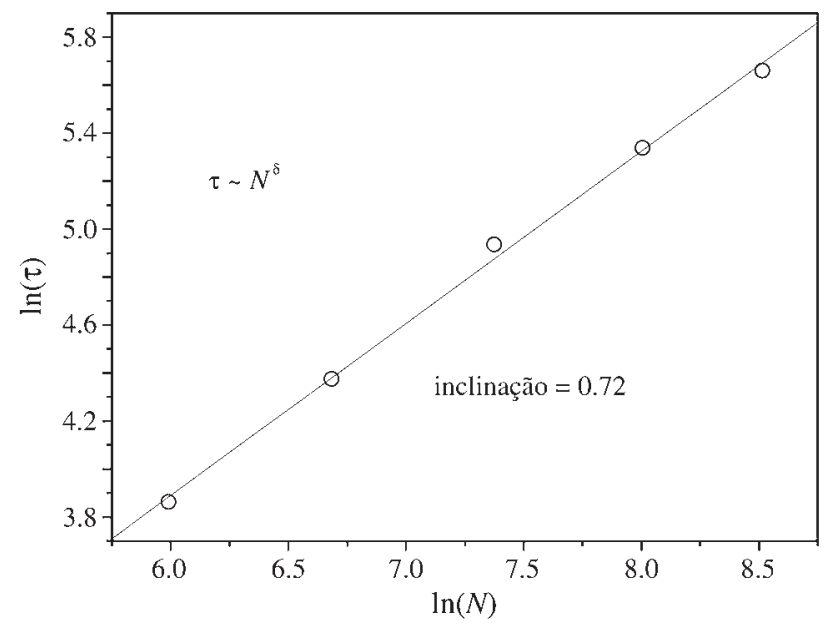

Figura 4 - Tempo de sobrevivência como função do número de sítios. Esta figura sugere que $\tau \sim N^{\delta}$.

de permanência em um estado ativo cresce com a conectividade e com o tamanho da rede, sugerindo que o sistema permanece, assintoticamente, em um estado desordenado independentemente do valor de $m$.

Durante nossas simulações avaliamos também o comportamento do tempo de sobrevivência $\tau$ com o tamanho da rede, para diferentes valores de conectividade. A Fig. 4 mostra a o tempo de sobrevivência para redes de escala livre com $m=2$ e diferentes tamanhos de rede. Podemos perceber que $\tau$ aumenta com o tamanho da rede segundo uma lei de potências $\left(\tau \sim \mathrm{N}^{\delta}\right)$, revelando que quanto maior o número de elementos da rede maior é a dificuldade do sistema em atingir um estado de ordenamento completo. Comportamento semelhante é observado também nas redes de maior conectividade média. $\mathrm{O}$ crescimento de $\tau$ como lei de potência revela que, para redes muito grandes, as interações de longo alcance mantém o sistema em um "estado transiente", independentemente da conectividade média da rede, ou seja, sugere que o sistema permanece assintoticamente em um estado desordenado.

\section{Conclusão}

Simulamos o modelo votante definido sobre redes de escala livre. Verificamos que o sistema permanece assintoticamente desordenado, ou seja, ao contrário do que ocorre em redes regulares o sistema não converge para um estado absorvente. Em redes de tamanhos finitos, o sistema permanece em um estado ativo durante um tempo $\tau$ após o qual converge para um estado totalmente ordenado. $\mathrm{O}$ tem- po médio de permanência em um estado ativo aumenta com o tamanho da rede. Nossos resultados estão de acordo com as simulações do modelo votante em redes Small World, que sugerem que conexões de longo alcance possuem influência direta sobre as propriedades de ordenamento do modelo, acelerando o processo de ordenamento, porém, de forma não trivial.

\section{Agradecimentos}

Agradecemos ao CNPq (Conselho Nacional de Desenvolvimento Científico e Tecnológico) pelo apoio financeiro através do PIBIC/UNIOESTE/CNPq.

\section{Referências}

[1] K. Binder, Monte Carlo Methods in Condensed Matter Physics (Springer, London, 1995), $2^{\text {a }}$ ed.

[2] M. Hennecke, Int. J. Mod. Phys. C 8, 207 (1997).

[3] B. Zheng, Int. J. Mod. Phys. B 12, 1419 (1998).

[4] D. Stauffer, J. Artificial Societies and Social Simulation 5, artigo n. 1 (2002). Disponível eletronicamente em jasss.soc. surrey.ac.uk.

[5] D. Stauffer, Int. J. Mod. Phys. C 13, 315 (2002).

[6] S.G. Alves, M.L. Martins e A.T. Bernardes, Ciência Hoje 32, 20 (2002).

[7] K. Sznajd-Weron e J. Sznajd, Int. J. Mod. Phys. C 11, 1057 (2000).

[8] G. Deffuant, D. Neau, F. Amblard e G. Weisbuch, Adv. Complex Syst. 3, 87 (2000); G. Deffuant, F. Amblard, G. Weisbuch e T. Faure, J. Artificial Societies and Social Simulation 5, n. 4, artigo n. 1 (2002). Disponível eletronicamente em jasss.soc.surrey.ac.uk.

[9] D. Stauffer e A.O. Souza, Dicretized opinion dynamics of Deffuant on scale-free networks ArXiv:Cond-mat/0310243 v. 1,10 de Outubro de 2003.

[10] D. Stauffer e H. Meyer-Ortmanns, Simulation of Consensus Model of Deffuant et al. on a Barabási-Albert Network ArXiv:Cond-mat/0308231 v. 2, 29 de Setembro de 2003. Disponível eletronicamente em http://xxx.lanl.gov.

[11] T.M. Ligget, Interacting Particle Systems (Springer-Verlag, New York, 1985).

[12] P.L. Krapivsky, Phys. Rev. A 45, 1067 (1992).

[13] C. Castellano, D. Vilone e A. Vespignani, Europhys. Lett. 63, 153 (2003).

[14] D. Vilone e C. Castellano, ArXiv:Cond-mat/0308366 v. 2, 27 de Janeiro de 2004. Disponível eletronicamente em http://xxx.lanl.gov.

[15] D.J. Watts e S.H. Strogatz, Nature 393, 440 (1998).

[16] D.J. Watts, Small Worlds: The Dynamics of Networks between Order and Randomness (Princeton University Press, Princeton, 1999).

[17] R. Albert e A-L. Barabási, Rev. Mod. Phys. 74, 46 (2002).

[18] R. Albert, H. Jeong e A-L. Barabási, Nature 406, 378 (2000). 